\title{
Fatores associados ao risco para doenças não transmissíveis em adultos brasileiros: estudo transversal de base populacional
}

\author{
Letícia Casado Costa* \\ Luiz Claudio Santos Thuler**
}

\begin{abstract}
No Brasil, devido às mudanças nos perfis demográfico, epidemiológico e nutricional da população, as doenças infecciosas vêm cedendo lugar às crônicodegenerativas. Em face da grande heterogeneidade demográfica, social e econômica do país, são exigidas ações de saúde pública que sejam direcionadas às realidades locais. Com o objetivo de identificar os fatores associados ao risco para doenças não transmissíveis em adultos brasileiros, foi realizado um inquérito domiciliar, entre junho de 2002 e outubro de 2005, em amostra representativa de 18 capitais brasileiras. As prevalências dos fatores de risco para doenças crônicas não transmissíveis foram: excesso de peso; atividade física irregular ou sedentarismo;consumo de cigarros; consumo de álcool de risco; hipertensão arterial referida; diabetes referida; e hipercolesterolemia referida. A ausência de fatores de risco foi observada em apenas $8,5 \%$ da população entrevistada. Conclui-se que a alta prevalência de exposição a pelo menos um dos fatores de risco estudados evidencia que as ações em saúde devem ser multifatoriais e não dirigidas somente a um fator isolado.
\end{abstract}

Palavras-chave: Fatores de risco. Doenças não transmissíveis. Prevalência. Estudos transversais. Brasil.

\section{Introdução}

As transformações sociais e econômicas pelas quais o Brasil vem passando têm causado mudanças relevantes no perfil de morbimortalidade da população. As doenças infecciosas e parasitárias, principais causas de morte no início do século passado, cederam lugar às doenças crônicas não transmissíveis (DCNTs) (CASADO; VIANNA; THULER, 2009). Essa transição epidemiológica tem se refletido na área de saúde pública e o desenvolvimento de estratégias para seu controle tornou-se uma emergência para o Sistema Único de Saúde (SUS).

Diante da constatação das mudanças ocorridas nos perfis demográfico, epidemiológico e nutricional da população brasileira e do aumento significativo da prevalência das DCNTs, o Ministério da Saúde (MS), com a intenção de monitorar essa nova realidade brasileira e diminuir a carga das doenças, baseou-se nas orientações da Organização Mundial da Saúde (OMS) e da Organização Pan-Americana de Saúde (Opas) e implan-

\footnotetext{
* Mestre em Neurologia, pelo Centro de Ciências Biológicas e da Saúde (CCBS) da Universidade Federal do Estado do Rio de Janeiro (Unirio), chefe do Serviço de Edição e Informação Técnico-Científica do Instituto Nacional de Câncer (Inca). ** Professor da pós-graduação stricto sensu do Instituto Nacional de Câncer (Inca) e da Universidade Federal do Estado do Rio de Janeiro (Unirio).
} 
tou um Sistema Nacional de Vigilância de Doenças e Agravos Não Transmissíveis (Dants). Entre outras ações, foi realizado o Inquérito Domiciliar sobre Comportamentos de Risco e Morbidade Referida de Doenças e Agravos Não Transmissíveis, que serviu como linha de base de informação para a constituição do Sistema de Doenças e Agravos Não Transmissíveis (Sisdant) e para o fortalecimento de redes nacionais de vigilância das doenças e agravos não transmissíveis (CASADO; VIANNA; THULER, 2009; INCA, 2004).

Ultimamente vem sendo cada vez mais usado o termo Dants para referir-se ao conjunto constituído pelas doenças crônicas não transmissíveis e pelos agravos (acidentes e violências). As doenças crônicas são definidas como afecções de saúde que acompanham os indivíduos por longo período de tempo, podendo apresentar momentos de piora (episódios agudos) ou melhora sensível. A vigilância epidemiológica das Dants deve reunir um conjunto de ações que possibilitem conhecer sua distribuição, magnitude e tendência de exposição aos fatores de risco na população, identificando seus condicionantes sociais, econômicos e ambientais, com o objetivo de subsidiar o planejamento, a execução e a avaliação das ações de prevenção e controle das mesmas, implementando assim políticas públicas voltadas para a promoção da saúde (BRASIL, 2011; CASADO; VIANNA; THULER, 2009). As DCNTs caracterizam-se por ter etiologia incerta, múltiplos fatores de risco, longos períodos de latência, curso prolongado, origem não infecciosa e por estarem associadas a deficiências e incapacidades funcionais (CASADO; VIANNA; THULER, 2009). Entre as mais importantes estão a hipertensão arterial, o diabetes, as neoplasias, as doenças cérebrovasculares e as doenças pulmonares obstrutivas crônicas (INCA, 2004).

Em função da heterogeneidade demográfica, social e econômica do Brasil, são observados diferentes padrões de mortalidade e morbidade por DCNTs nas diferentes regiões, exigindo ações que sejam adequadas à realidade encontrada
(CASADO; VIANNA; THULER, 2009; INCA, 2004). Devido à necessidade de informações mais detalhadas sobre os fatores de risco para as DCNTs, principalmente em localidades ainda não estudadas, justificase a realização do presente estudo que tem por objetivo traçar o perfil epidemiológico da população de 25 anos e mais em relação à exposição e à presença de comportamentos de risco conhecidos e modificáveis em cidades brasileiras. Os resultados poderão servir de subsídio para o planejamento e gestão da saúde nas diferentes regiões, visando a melhoria da qualidade das ações dirigidas à população.

\section{Materiais e métodos}

Foram analisados dados extraídos do Inquérito Domiciliar sobre Comportamentos de Risco e Morbidade Referida de Doenças e Agravos Não Transmissíveis, levado a campo entre 2002 e 2005. Trata-se de um estudo transversal de base populacional, cujos detalhes metodológicos referentes à primeira fase, realizada entre 2002 e 2003, encontram-se em publicação elaborada pelo MS (INCA, 2004). No atual estudo foram incluídas as 18 cidades brasileiras participantes (Manaus, Belém, Palmas, São Luis, Fortaleza, Natal, João Pessoa, Recife, Aracaju, Vitória, Rio de Janeiro, Belo Horizonte, São Paulo, Curitiba, Florianópolis, Porto Alegre, Campo Grande e Brasília), contemplando-se somente os dados referentes à população adulta de 25 anos ou mais e agregando-se novos componentes analíticos.

Amostras por conglomerados, com dois estágios de seleção (setores censitários e domicílios), e autoponderadas foram utilizadas para cada município. A seleção dos setores foi feita de forma sistemática e proporcional ao total de domicílios particulares. Em cada setor selecionado elaborou-se o cadastro de todos os domicílios, classificando-os em ocupado, fechado, vago, uso ocasional e não residencial. Posteriormente, foram selecionados os domicílios também de forma sistemática.

Para determinação do tamanho da amostra, para cada município, consideraram- 
se um grau de confiabilidade de 95\%, uma precisão relativa de $10 \%$ e a prevalência estimada do evento estudado de $27 \%$ (BRASIL, 2011)(única estimativa nacional da prevalência de fumantes conhecida até então). Este último valor teve por objetivo determinar com precisão a prevalência do tabagismo, um dos fatores de risco de maior importância epidemiológica para doenças não transmissíveis.

Para a amostra das 18 cidades, foram selecionados 1.048 setores censitários. No total, foram visitados 13.885 domicílios, sendo que $12.375(89,1 \%)$ eram ocupados e $1.510(10,9 \%)$ não ocupados. Entre os ocupados, foi possível fazer entrevista em $11.323(91,5 \%)$. Não foram estudados 1.052 $(8,5 \%)$ domicílios ocupados devido aos mesmos estarem fechados, haver recusas ou outro motivo. A amostra da população elegível oriunda dos domicílios entrevistados correspondeu a 21.251 pessoas, porém 1.999 indivíduos não foram incluídos na pesquisa por ausência no dia da entrevista, doença ou recusa.

Para este estudo, as informações sobre as características sociodemográficas da população estudada foram extraídas do questionário domiciliar, enquanto aquelas referentes aos comportamentos de risco foram coletadas do questionário para adulto. Os questionários foram elaborados com base em instrumentos validados (Standard Risk Factor Questionnaire, da Organização Mundial da Saúde, Behavioral Risk Factor Surveillance System, dos Centros de Controle de Doenças dos Estados Unidos da América, Third Health and Nutrition Examination Survey, do Departamento de Saúde dos Estados Unidos da América, Projeto Carmen - Organização Mundial da Saúde/Organização Panamericana da Saúde, e International Physical Activity Questionnaire Young and Middle-aged Adults, do Instituto Karolinska da Suécia) e foram testados antes de sua utilização.

Um estudo-piloto, realizado no Município do Rio de Janeiro em maio de 2002, com a aplicação de 60 questionários nos bairros de Bangu, Botafogo e Grajaú, possibilitou a revisão do questionário e a definição da logística do trabalho de campo.
As entrevistas foram realizadas por uma equipe treinada composta por entrevistadores do sexo feminino, supervisores e um coordenador do trabalho de campo, selecionados em cada localidade com o apoio das Secretarias de Saúde e do IBGE local. Os supervisores revisitaram todos os domicílios não entrevistados e uma amostra de $30,0 \%$ daqueles entrevistados, com o objetivo de garantir a qualidade das informações.

\section{Variáveis de estudo}

Foram estudadas as seguintes variáveis: idade (em anos completos na data da entrevista, categorizada em quatro faixas - menos de 40, 40 a 59, 60 a 79 e 80 anos e mais); situação conjugal (casado, divorciado/separado, solteiro e viúvo); escolaridade (em anos de estudo, categorizada em duas faixas - fundamental incompleto menos de oito anos de estudo e fundamental completo oito e mais anos de estudo); situação de trabalho (com trabalho e sem trabalho); faixa de renda per capita (soma das rendas dos integrantes da família principal dividida pelo total de moradores do domicílio, em salários mínimos); região de residência (Norte, Nordeste, Sudeste, Sul e Centro-Oeste); peso (utilizou-se o Índice de Massa Corporal - IMC a partir da informação do peso e da estatura referidos); atividade física (os indivíduos foram classificados como: muito ativos, ativos e insuficientemente ativos); tabagismo (não fumante, ex-fumante e fumante); consumo de álcool (foi considerada de risco a ingestão de doses diárias acima de uma dose/dia para as mulheres e duas doses/dia para os homens); hipertensão arterial (referida); diabetes (referida); hipercolesterolemia (referida) (INCA, 2004).

Para investigar a aglomeração de pelo menos um fator de risco para DCNTs, foi criado um escore que reuniu os fatores de risco modificáveis (excesso de peso: sobrepeso + obesidade; atividade física: insuficientemente ativa $=$ irregularmente ativa + sedentária; tabagismo e consumo de álcool de risco) e morbidade referida (hipertensão, diabetes e hipercolesterolemia). A informação sobre atividade física foi restrita à população de até 69 anos. 


\section{Análise estatística}

Foi desenvolvido um aplicativo de informática em linguagem Delphi com utilização da plataforma Oracle para digitação dos dados. Para controlar a qualidade da digitação, empregou-se um dígito verificador que consistiu no somatório dos números de quadrículas assinaladas ou preenchidas em cada página do questionário.

As estimativas de proporção e de associação e os respectivos erros-padrão foram calculados considerando o plano de amostragem por conglomerados. Foi utilizado o programa estatístico Stata 8.0 (2003), que por meio do módulo "survey" permite a análise de dados provenientes de inquéritos epidemiológicos com estratégias complexas de amostragem.

Inicialmente, foi feita uma caracterização da amostra em relação às variáveis e, posteriormente, foram calculados as proporções e seus respectivos intervalos com 95\% de confiança $\left(\mathrm{IC}_{95 \%}\right)$.

Com o intuito de estudar a associação entre a presença de pelo menos um fator de risco para DCNTs e algumas características sociodemográficas selecionadas, foram calculadas razões de prevalência por meio de Regressão de Poisson. Valores de $p$ menor que 0,05 foram considerados estatisticamente significantes.

\section{Considerações éticas}

O estudo foi desenvolvido de forma a proteger a privacidade dos indivíduos, garantindo a participação anônima e voluntária. Um termo de consentimento livre e esclarecido assinado pelo informante era uma exigência para a participação. O estudo foi aprovado pela Comissão de Ética e Pesquisa do Instituto Nacional de Câncer (Inca/ MS), sendo respeitadas as recomendações do Conselho Nacional de Ética em Pesquisa (Conep).

\section{Resultados}

Foram entrevistados 8.266 homens e 10.986 mulheres. Os respondentes eram majoritariamente do sexo feminino $(57,8 \%)$, com menos de 40 anos de idade (44,0\%) e referiram ser casados ou ter união estável $(65,2 \%)$. Cerca de $60 \%$ dos respondentes tinham o ensino fundamental completo ou mais e 38\% não trabalhavam (Tabela 1).

$\mathrm{Na}$ Tabela 2, estão descritas as prevalências dos fatores de risco estudados nas 18 capitais brasileiras. Como pode ser visto, o excesso de peso (sobrepeso + obesidade) apresentou a maior prevalência $(48,1 \%)$. Já $39,7 \%$ da população pesquisada foi classificada como insuficientemente ativa (irregularmente ativa + sedentária). Entre os entrevistados, $20,8 \%$ mencionaram consumo de cigarros e, para $7,8 \%$, registrou-se consumo elevado de álcool. Entre os adultos, $25,2 \%$ afirmaram ser hipertensos e $8,0 \%$ diabéticos, enquanto a hipercolesterolemia foi mencionada por $23,6 \%$. A ausência de fatores de risco foi observada em apenas $8,5 \%$ da população.

Quanto ao estado de saúde autorreferido, observou-se que 15,3\% (IC 95\% 14,4-16,4) dos entrevistados o consideraram excelente $(18,7 \%$ homens e $12,9 \%$ mulheres); $16,3 \%$ (IC 95\% 15,2-17,5) referiram como muito bom $(17,4 \%$ do sexo masculino e $15,5 \%$ do feminino); $40,2 \%$ (IC $95 \% 38,8-$ $41,5)$ como bom $(40,6 \%$ homens e $39,9 \%$ mulheres); 24,4\% (IC 95\% 23,1-25,7) como regular (20,4\% homens e $27,3 \%$ mulheres); e $3,8 \%$ (IC 95\% 3,3-4,4) como ruim $(3,0 \%$ homens e $4,4 \%$ mulheres).

A Tabela 3 apresenta os fatores associados à presença de pelo menos um fator de risco na população estudada. A razão de prevalências aponta para a aglomeração de um ou mais fatores de risco entre pessoas do sexo masculino, com 60 anos e mais, com menor escolaridade, que não têm trabalho, com renda per capita inferior a um salário mínimo e cuja autoavaliação do estado de saúde foi regular ou ruim $(p<0,05)$. A situação conjugal e o local de residência não estiveram associados à presença de pelo menos um fator de risco para as DCNTs $(p>0,05)$.

\section{Discussão}

Atualmente, as DCNTs representam uma grande preocupação na área da saúde pública, sendo responsáveis por uma 
TABELA 1

População de 25 anos e mais, segundo características socioeconômicas e demográficas Capitais brasileiras (1) - 2002-2005

\begin{tabular}{|c|c|c|}
\hline Características sociodemográficas & N. abs. & $\%$ (IC 95\%) (2) \\
\hline \multicolumn{3}{|l|}{ Sexo } \\
\hline Homens & 8.266 & $42,2(41,3-43,1)$ \\
\hline Mulheres & 10.986 & $57,8(56,9-58,7)$ \\
\hline \multicolumn{3}{|l|}{ Faixa etária } \\
\hline Menos de 40 anos & 8.856 & $44,0(42.5-45.5)$ \\
\hline $40-59$ anos & 7.448 & $40,3(39,0-41,6)$ \\
\hline 60-79 anos & 2.667 & $14,3(13,4-15,2)$ \\
\hline 80 anos e + & 281 & $1,5(1,2-1,8)$ \\
\hline \multicolumn{3}{|l|}{ Situação conjugal } \\
\hline Casado & 12.657 & $65,2(63,7-67,0)$ \\
\hline Divorciado/separado & 1.939 & $10,1(9,4-10,9)$ \\
\hline Solteiro & 3.396 & $17,8(16,7-18,9)$ \\
\hline Viúvo & 1.259 & $6,9(6,3-7,6)$ \\
\hline \multicolumn{3}{|l|}{ Escolaridade } \\
\hline Fundamental incompleto & 7.854 & $42,0(39,7-44,0)$ \\
\hline Fundamental completo ou mais & 11.350 & $58,1(56,0-60,3)$ \\
\hline \multicolumn{3}{|l|}{ Situação de trabalho } \\
\hline Com trabalho & 12.045 & $62,0(60,6-63,3)$ \\
\hline Sem trabalho & 7.206 & $38,0(36,7-39,4)$ \\
\hline \multicolumn{3}{|l|}{ Faixa de renda per capita } \\
\hline Menos de um salário mínimo & 8.038 & $39,4(36,8-42,1)$ \\
\hline 1-3 salários mínimos & 5.758 & $34,1(32,0-36,2)$ \\
\hline 3-5 salários mínimos & 1.770 & $10,3(9,1-11,6)$ \\
\hline Mais de 5 salários mínimos & 2.379 & $14,9(13,1-16,9)$ \\
\hline \multicolumn{3}{|l|}{ Região de residência } \\
\hline Norte & 2.958 & $7,0(5,7-8,6)$ \\
\hline Nordeste & 5.594 & $18,4(16,1-21,0)$ \\
\hline Sudeste & 5.303 & $57,6(53,5-61,5)$ \\
\hline Sul & 3.508 & $9,5(8,1-11,1)$ \\
\hline Centro-Oeste & 1.889 & $7,5(5,9-9,4)$ \\
\hline
\end{tabular}

Fonte: Ministério da Saúde. Inquérito Domiciliar sobre Comportamentos de Risco e Morbidade Referida de Doenças e Agravos Não Transmissíveis.

(1) Compreendem 18 capitais do Brasil.

(2) IC = Intervalo de confiança.

TABELA 2

População de 25 anos ou mais, por sexo, segundo fatores de risco para DCNTs Capitais brasileiras (1) - 2002-2005

\begin{tabular}{lrrr}
\hline Fatores de risco & \multicolumn{1}{c}{$\begin{array}{c}\text { Total } \\
\text { (IC 95\%) (2) }\end{array}$} & $\begin{array}{c}\text { Homens } \\
\text { (IC 95\%)(2) }\end{array}$ & \multicolumn{1}{c}{$\begin{array}{c}\text { Mulheres } \\
\text { (IC 95\%) (2) }\end{array}$} \\
\hline Excesso de peso & $48,1(46,6-49,6)$ & $53,9(51,8-55,9)$ & $43,1(41,2-45,2)$ \\
Insuficientemente ativo & $39,7(38,0-41,5)$ & $35,9(33,7-38,1)$ & $42,6(40,5-44,7)$ \\
Tabagismo & $20,8(19,8-21,9)$ & $25,2(23,6-26,8)$ & $17,7(16,4-19,1)$ \\
Consumo de álcool de risco & $7,8(7,2-8,5)$ & $13,0(11,9-14,3)$ & $4,0(3,5-4,5)$ \\
Hipertensão arterial & $25,2(24,1-26,4)$ & $22,8(21,2-24,4)$ & $28,6(27,1-30,2)$ \\
Diabetes & $8,0(7,1-8,9)$ & $9,4(7,9-11,1)$ & $7,1(6,2-8,2)$ \\
Hipercolesterolemia & $23,6(22,4-24,8)$ & $22,0(20,2-23,9)$ & $24,6(23,0-26,1)$ \\
\hline
\end{tabular}

Fonte: Ministério da Saúde. Inquérito Domiciliar sobre Comportamentos de Risco e Morbidade Referida de Doenças e Agravos Não Transmissíveis.

(1) Compreendem 18 capitais do Brasil.

(2) IC = Intervalo de confiança 
TABELA 3

Associação entre características sociodemográficas e a presença de pelo menos um fator de risco para DCNTs para a população de 25 anos ou mais

Capitais brasileiras (1) - 2002-2005

\begin{tabular}{|c|c|c|c|c|c|}
\hline $\begin{array}{l}\text { Características } \\
\text { sociodemográficas }\end{array}$ & $\begin{array}{l}\text { Sem fator de } \\
\text { risco }(\%) \\
n=929\end{array}$ & $\begin{array}{l}\text { Um ou mais fatores } \\
\text { de risco (\%) } \\
n=10.083\end{array}$ & $\begin{array}{c}\text { Razão de } \\
\text { prevalência }\end{array}$ & IC 95\% (2) & Valor de $p$ \\
\hline \multicolumn{6}{|l|}{ Sexo } \\
\hline Homens & 6,4 & 93,6 & 1,04 & $(1,02-1,05)$ & $\mathrm{p}<0,001$ \\
\hline Mulheres & 10,0 & 90,0 & 1 & ref. (3) & \\
\hline \multicolumn{6}{|l|}{ Faixa etária } \\
\hline Até 60 anos & 5,1 & 94,9 & 1,04 & $(1,02-1,06)$ & $\mathrm{p}<0,001$ \\
\hline Mais de 60 anos & 9,1 & 90,9 & 1 & ref. (3) & \\
\hline \multicolumn{6}{|l|}{ Situação conjugal } \\
\hline Casado & 8,0 & 92,0 & 1,01 & $(0,99-1,03)$ & $p=0,1288$ \\
\hline Outra situação & 9,3 & 90,7 & 1 & ref. (3) & \\
\hline \multicolumn{6}{|l|}{ Escolaridade } \\
\hline Fundamental incompleto & 4,0 & 96,0 & 1,08 & $(1,07-1,10)$ & $\mathrm{p}<0,001$ \\
\hline Fundamental completo ou mais & 11,6 & 88,4 & 1 & ref. (3) & \\
\hline \multicolumn{6}{|l|}{ Situação de trabalho } \\
\hline Sem trabalho & 6,0 & 94,0 & 1,04 & $(1,02-1,06)$ & $\mathrm{p}<0,001$ \\
\hline Com trabalho & 10,0 & 90,0 & 1 & ref. (3) & \\
\hline \multicolumn{6}{|l|}{ Faixa de renda per capita } \\
\hline Menos de um salário mínimo & 5,2 & 94,8 & 1 & ref. (3) & $\mathrm{p}<0,001$ \\
\hline 1-3 salários mínimos & 8,1 & 91,9 & 0,97 & $(0,95-0,99)$ & \\
\hline 3-5 salários mínimos & 12,5 & 87,5 & 0,92 & $(0,89-0,96)$ & \\
\hline Mais de 5 salários mínimos & 13,4 & 86,6 & 0,91 & $(0,88-0,94)$ & \\
\hline \multicolumn{6}{|l|}{ Região de residência } \\
\hline Sudeste & 8,9 & 91,1 & 0,98 & $(0,97-1,00)$ & $p=0,1069$ \\
\hline Outras regiões & 7,8 & 92,2 & 1 & ref. (3) & \\
\hline \multicolumn{6}{|l|}{ Estado de saúde regular ou ruim } \\
\hline $\operatorname{Sim}$ & 4,0 & 96,0 & 1,07 & $(1,05-1,08)$ & $p<0,001$ \\
\hline Não & 10,3 & 89,7 & 1 & ref. (3) & \\
\hline
\end{tabular}

Fonte: Ministério da Saúde. Inquérito Domiciliar sobre Comportamentos de Risco e Morbidade Referida de Doenças e Agravos Não Transmissíveis

(1) Compreendem 18 capitais do Brasil.

(2) IC = Intervalo de confiança.

(3) ref. = valor de referência.

parcela significante e crescente da carga de doenças e por cerca de $60 \%$ dos óbitos no Brasil (SOUZA, 2008).

Para que seja possível monitorar os comportamentos de risco que levam a população a desenvolver DCNTs, é necessário conhecer o perfil de exposição populacional aos seus fatores de risco, ou seja, estilos de vida, hábitos, exposições e comorbidades. Os estudos brasileiros sobre fatores de risco para DCNTs são recentes e em pequeno número. A maioria concentra-se na Região Sudeste do país. Somente três trabalhos apresentaram resultados para as capitais dos Estados brasileiros e o DF (BRASIL, 2008, 2009; MONTEIRO et al., 2005), mesmo assim com algumas limitações por serem inquéritos telefônicos. $O$ presente estudo realizado no Brasil, entre 2002 e 2005, dedicou-se a suprir essa lacuna do conhecimento ao estudar a prevalência de fatores de risco para as DCNTs na população de 18 capitais.

No que se refere à prevalência de excesso de peso, o resultado encontrado neste estudo foi elevado $(48,1 \%)$. Essa 
observação está de acordo com aquelas assinaladas em outros estudos (CARVALHAES; MOURA; MONTEIRO, 2008; COSTA et al., 2004; CUNHA et al., 2008; MONTEIRO et al., 2005; PEIXOTO et al., 2008; YOKOTA et al., 2007)que, de modo geral, indicam prevalências que variam entre $40 \%$ e $50 \%$. A pequena variabilidade nessas proporções pode dever-se ao fato de haver consenso quanto à padronização do cálculo do IMC. Em todas as cidades analisadas, o excesso de peso foi mais frequente em homens do que em mulheres, o que está de acordo com os dados encontrados por outros autores (CARVALHAES; MOURA; MONTEIRO, 2008; CUNHA et al., 2008; MONTEIRO et al., 2005; MOURA, 2008; PEIXOTO et al., 2008).

A prática regular de atividades físicas entre os níveis moderados e vigorosos funciona como fator de proteção para morbimortalidade por doenças cardiovasculares e obesidade. É fundamental a identificação dos determinantes desse comportamento para serem propostas estratégias populacionais com vista à adoção de um estilo de vida fisicamente ativo. Estimativas globais da OMS (WHO, 2002) indicam que a inatividade física é responsável por quase dois milhões de mortes, por $22 \%$ dos casos de doença isquêmica do coração e por $10 \%$ a $16 \%$ dos casos de diabetes e de cânceres de mama e cólon e reto. Dados divulgados pela Sociedade Brasileira de Cardiologia (SBC) apontam que $80 \%$ da população brasileira adulta é sedentária.

O presente estudo estimou a prevalência de insuficientemente ativos a partir da soma daqueles que mencionaram ser sedentários e os irregularmente ativos. Analisando a população total do estudo, observou-se que quase $40 \%$ dos entrevistados encontravam-se nessa situação. Esse comportamento foi mais frequente em mulheres $(42,6 \%)$ do que em homens $(35,9 \%)$, sendo essa diferença estatisticamente significativa. Esses valores foram semelhantes àqueles observados em mulheres $(58,2 \%)$ no estudo de Carvalhaes (2008). Por fim, as diferenças entre as prevalências identificadas no presente estudo e aquela obtida pelos diferentes autores podem dever-se à forma como essa variável foi por eles classificada: dias por semana com atividade física no lazer (pelo menos 30 minutos) (CARVALHAES; MOURA; MONTEIRO, 2008; CUNHA et al., 2008); atividade física ocupacional (CUNHA et al., 2008); atividade física no deslocamento (CARVALHAES; MOURA; MONTEIRO, 2008; CUNHA et al., 2008); atividade física na limpeza (CUNHA et al., 2008); fisicamente inativos (sem prática de qualquer atividade física no lazer nos últimos três meses e que não realizam esforços físicos intensos no trabalho, não se deslocam para o trabalho caminhando ou de bicicleta e não são responsáveis pela limpeza pesada de suas casas) (CARVALHAES; MOURA; MONTEIRO, 2008); e sedentarismo (CARVALHAES; MOURA; MONTEIRO, 2008; CUNHA et al., 2008).

Apesar de alta, a prevalência de $20,8 \%$ de fumantes de cigarros encontrada no presente estudo foi inferior àquela observada na Pesquisa Nacional de Saúde e Nutrição, realizada em 1989 pelo IBGE $(30,2 \%)$. Mais recentemente, em sua terceira fase, o Vigitel 2008 (BRASIL, 2009) encontrou prevalência de $15,2 \%$ de fumantes entre os brasileiros. Embora as metodologias dos estudos não sejam comparáveis, os resultados indicam uma expressiva redução nesses percentuais. Pode-se considerar, como um dos responsáveis por esse declínio no Brasil, a implantação de ações de impacto (legislação, advertências nos produtos sobre os malefícios do tabagismo, criação e ampliação dos ambientes livres do tabaco e restrição ou proibição da propaganda), coordenadas pelo Inca/MS, a partir do Programa Nacional de Controle do Tabagismo, que, do ponto de vista cronológico, podem estar refletidas na redução desse percentual. Esses achados podem ser considerados um importante indício de que as políticas públicas de controle do tabaco vêm obtendo êxito (FIGUEIREDO, 2008). Também nos Estados Unidos, o Centers for Disease Control and Prevention (2008) fez uma comparação da prevalência anual de tabagismo entre adultos norte-americanos, verificando uma redução de $24,7 \%$, em 1997, para 19,7\%, em 2007, entre os fumantes.

Quanto ao perfil do usuário, neste estudo o comportamento de fumar mostrou-se 
mais frequente entre homens do que entre mulheres em todas as regiões do país, embora as diferenças segundo gênero tenham variado muito de cidade para cidade. Estes resultados também concordam com os apontados em outros estudos (BRASIL, 2008, 2009), que, de modo geral, indicam uma prevalência maior entre os homens.

A literatura científica indica que o consumo moderado de bebidas alcoólicas é um fator de proteção para mortalidade por DCNTs, principalmente por seu efeito redutor sobre as doenças cardiovasculares. Já o consumo abusivo traz inúmeras consequências para a saúde e qualidade de vida, aumentando a frequência de morbidades que causam morte ou limitações funcionais, como a cirrose, alguns tipos de câncer, acidente vascular cerebral, violência, transtorno mental, entre outros.

A dependência química do álcool, atualmente chamada de alcoolismo, por si só, também é considerada uma doença (SOUZA et al., 2003). Este estudo classificou como de risco o consumo diário acima do limite recomendado pela OMS: mais de duas doses padronizadas de bebida alcoólica para homens e mais de uma dose padronizada para mulheres (WHO, 2000). No conjunto da população adulta das 18 capitais estudadas, a frequência do consumo de risco de bebidas alcoólicas foi de $7,8 \%$, sendo três vezes maior em homens $(13,0 \%)$ do que em mulheres $(4,0 \%)$, o que é consistente com a literatura científica (CARVALHAES; MOURA; MONTEIRO, 2008; COSTA et al., 2004; LESSA, 2004; MONTEIRO et al., 2005; MOURA, 2008; PEIXOTO et al., 2008; PEREIRA; BARRETO; PASSOS, 2008), que mostra prevalências que variam de $0,1 \%$ a $37,7 \%$. Essas diferenças podem ser, em parte, devidas ao fato de não ter sido usado um questionário padronizado para avaliação desse fator de risco. Os resultados deste estudo indicam ainda que os homens constituem o grupo com maior consumo abusivo de álcool e, portanto, os mais suscetíveis às suas consequências.

Entre os fatores de risco modificáveis, a hipertensão arterial é considerada o mais importante para a morbimortalidade por DCNTs, especialmente no que diz respeito às doenças isquêmicas e ao acidente vascular cerebral. O interesse em obter informações sobre a prevalência de hipertensão arterial referida deveu-se aos custos e complexidade da medida objetiva da pressão arterial. Apesar das limitações, fornecem informações úteis para avaliar a demanda por cuidados de saúde originada pela sua presença. É evidente que as frequências estimadas dessa maneira serão influenciadas pela cobertura da assistência à saúde existente em cada localidade, podendo, assim, subestimar, em maior ou menor grau, a prevalência real desse fator de risco na população.

As altas prevalências de pessoas que disseram ter pressão arterial elevada neste estudo, em ambos os sexos, concordaram com os dados do Vigitel 2008 (BRASIL, 2009), cuja frequência de diagnóstico médico prévio de hipertensão arterial alcançou $23,1 \%$, sendo ligeiramente maior em muIheres (25,5\%) do que em homens (20,3\%).

Segundo vários autores (CARVALHAES; MOURA; MONTEIRO, 2008; COSTA et al., 2004; CUNHA et al., 2008; LESSA, 2004; MARCOPITO et al., 2005; MONTEIRO et al., 2005; PEIXOTO et al., 2008; PEREIRA; BARRETO; PASSOS, 2008; SOUZA et al., 2003; YOKOTA et al., 2007), mesmo levando-se em conta as diferenças metodológicas entre os estudos que dificultam uma abordagem conjunta dos achados, sua prevalência nas diferentes regiões do país vem se mostrando elevada. De acordo com a literatura científica, as prevalências variam entre 10,9\% (CUNHA et al., 2008) e 50,6\% (PEREIRA; BARRETO; PASSOS, 2008) e dependem, principalmente, da população estudada e dos métodos utilizados na definição e aferição da pressão arterial. Diferenças podem ainda ser explicadas pelo ponto de corte para classificação dos níveis tensionais, que mudou nos últimos anos, de acordo com a Classificação de Pressão Arterial da Sociedade Brasileira de Hipertensão (2011), levando à baixa prevalência em algumas cidades, em função do ano de coleta de dados. Isso foi observado no resultado de Souza et al. (2003), que se basearam nos critérios definidos pelo VI Joint National Committe de 1997. 
Um aumento no número de casos de diabetes vem sendo observado em vários países em desenvolvimento e tem como determinantes as transições demográficas e nutricionais em curso. O conhecimento das estimativas de prevalência dessa morbidade é fundamental, a fim de que seja possível estabelecer políticas públicas específicas de prevenção e atenção à saúde. Até 2002, no Brasil, só existia informação sobre a prevalência de diabetes proveniente de um único inquérito populacional, realizado no final da década de 1980, em nove capitais (SOCIEDADE BRASILEIRA DE DIABETES, 2009). O presente estudo ocorreu 14 anos após a realização do primeiro, possibilitando assim um conhecimento mais atual dessa patologia, além de permitir estimativas deste agravo em cidades onde a prevalência era completamente desconhecida.

No conjunto da população adulta das 18 cidades estudadas, a frequência do diagnóstico médico prévio de diabetes foi maior em homens $(9,4 \%)$ do que em mulheres $(7,1 \%)$. Esses resultados diferem daqueles descritos no Vigitel 2008 (BRASIL, 2009), no qual o índice da doença foi mais elevado em mulheres $(5,6 \%)$ do que em homens $(4,6 \%)$. Nos Estados Unidos, onde sua incidência é elevada, o diabetes afeta cerca de 23,6 milhões de pessoas, o que significa $8 \%$ da população (CENTERS FOR DISEASE CONTROL AND PREVENTION, 2007).

Entre junho e agosto de 2002, a Campanha Nacional de Alerta Sobre o Colesterol Elevado, por iniciativa do Departamento de Aterosclerose e da SBC/Funcor, mensurou os níveis sanguíneos de colesterol em 81.262 voluntários residentes nas cidades de Porto Alegre (RS), Curitiba (PR), São Paulo (SP), Rio de Janeiro (RJ), Belo Horizonte (MG), Salvador (BA), Brasília (DF), Campinas (SP), Campos do Jordão (SP), São José dos Campos (SP), Santos (SP), Santo André (SP) e Ribeirão Preto (SP). A partir do preenchimento de um questionário, o objetivo principal foi, além de identificar outros fatores de risco (como antecedentes familiares de doença arterial coronariana, diabetes, hipertensão arterial sistêmica e tabagismo), alertar a população sobre as necessidades de prevenir a doença cardiovascular. A medi- da foi determinada em voluntários e não em uma amostra representativa da população. O estudo concluiu que $40 \%$ da população total estudada apresentava níveis sanguíneos de colesterol elevado (MARTINEZ et al., 2003). A utilização de dados de morbidade referida, em vez da coleta objetiva, permite obter informações de maneira confiável a um custo mais adequado num país com a dimensão do Brasil (THEME FILHA; SZWARCWALD; SOUZA JUNIOR, 2008).

Os achados obtidos na prevalência de dislipidemia, maior entre as mulheres $(24,6 \%)$ do que entre os homens $(22,0 \%)$, corroboram os descritos no Vigitel 2008 (19,2\% para as mulheres e $14,0 \%$ para os homens) e no Simtel Goiânia 2005 (PEIXOTO et al., 2008)(21\% para as mulheres e $14,1 \%$ para os homens). É importante destacar que medidas feitas em voluntários tendem a superestimar a prevalência de fatores como o colesterol, pois é provável que pessoas com hábitos alimentares inadequados ou tendência familiar para hipercolesterolemia sintam-se mais estimuladas a participar e ter seu colesterol medido do que as demais (MARTINEZ et al., 2003). Comparações com os resultados de outros estudos brasileiros são difíceis de serem feitas porque as estatísticas disponíveis foram obtidas em diferentes áreas urbanas, em diversos pontos do tempo e usando diferentes metodologias.

Segundo a OMS, desde 1992, a autoavaliação do estado de saúde é considerada um dos indicadores mais frequentemente utilizados para se conhecer a percepção de saúde da população. Vários estudos revelam índices de $80 \%$ de concordância entre a autoavaliação do estado de saúde e a avaliação clínica da presença ou ausência de condição crônica. Outra questão que reforça sua importância é por ser um dado facilmente coletado (VIACAVA, 2002), o que o leva a ser amplamente utilizado em inquéritos de saúde. Essa autoavaliação do estado de saúde é obtida, habitualmente, por meio de uma única questão, que pede para o indivíduo classificar seu estado de saúde em excelente, bom, regular ou ruim. Neste inquérito, cada entrevistado avaliou seu próprio estado de saúde, diferentemente do que faz a Pesquisa Nacional por Amostra de 
Domicílio (PNAD), por exemplo, quando um entrevistado avaliou esse indicador para os demais moradores do domicílio (BARROS et al., 2006). Ao analisar esse indicador de saúde, foram encontradas autoavaliações piores entre as mulheres (31,9\%).

Segundo dados do National Health Interview Survey (NHIS), nos Estados Unidos, em 2002, o percentual de indivíduos que autoavaliaram seu estado de saúde como regular ou ruim foi de $12,2 \%$ (11,9\% entre homens e $12,6 \%$ entre mulheres). Em linhas gerais, com respeito ao estado de saúde referido, as mulheres declararam pior estado em ambos os países $(12,6 \%$ nos Estados Unidos e 31,9\% no Brasil), estando os brasileiros em pior situação.

Finalmente, no que diz respeito à presença de pelo menos um fator de risco para DCNTs, estudo realizado por Martinez et al. (2003) mostrou que a distribuição de pessoas sem fatores de risco para DCNTs $(30 \%)$, com um $(36 \%)$ e com dois ou mais fatores (34\%) foi semelhante. Já um inquérito realizado na população dos Estados Unidos, em 2001 , mostrou que $17 \%$ dos americanos apresentaram três ou mais fatores de risco para DCNTs. No presente estudo, mais de 90\% dos entrevistados apresentaram pelo menos um fator de risco.

A interpretação dos resultados desta pesquisa deve ser feita à luz de algumas limitações metodológicas. A primeira delas diz respeito à morbidade referida, que pode ter subestimado algumas prevalências. Mesmo considerando que a validade da informação referida é um limitante para as estimativas de prevalências de doenças crônicas, os padrões epidemiológicos observados estiveram, em sua maior parte, alinhados com os obtidos a partir de outras fontes

\section{Referências}

BARROS, M. B. A. et al. Desigualdades sociais na prevalência de doenças crônicas no Brasil, PNAD-2003. Ciência \& Saúde Coletiva, Rio de Janeiro, v. 11, n. 4, p. 911-26, 2006.

BRASIL. Ministério da Saúde. Secretaria de Vigilância em Saúde. Secretaria de de dados. Isso vem fazendo com que os inquéritos de saúde sejam crescentemente utilizados para avaliar o estado de saúde das populações e a ocorrência de morbidades. Inquéritos domiciliares têm alto custo, o que se deve a vários fatores. Um deles é o difícil acesso às áreas de risco nos grandes centros urbanos. Outra dificuldade está em encontrar as pessoas inicialmente selecionadas. Muitos indivíduos economicamente ativos e responsáveis pelo sustento da família não foram encontrados na primeira visita; algumas visitas foram remarcadas para ocorrer à noite, quando não se tratava de área de risco social.

Alguns aspectos metodológicos importantes a serem ressaltados são o modelo de amostragem adotado, os tamanhos das amostras, a propriedade dos instrumentos de coleta e o controle de qualidade do trabalho de campo, da digitação e da análise dos dados.

O presente estudo permitiu traçar um painel do perfil epidemiológico da prevalência de fatores de risco para doenças crônicas da população brasileira. Foram observadas significativas desigualdades na magnitude das prevalências e no perfil de morbidades. Muitas das diferenças são concordantes com resultados de outros inquéritos e outras fontes de dados. Conclui-se que as altas prevalências obtidas no estudo elaborado indicam a necessidade de realização de inquéritos populacionais periódicos e com intervalos regulares e estruturação de programas de intervenções visando a implementação de estratégias de prevenção e promoção da saúde para melhorar o perfil dos brasileiros, no que diz respeito à exposição aos fatores de risco para as DCNTs.

Gestão Estratégica e Participativa. Vigitel Brasil 2007: vigilância de fatores de risco e proteção para doenças crônicas por inquérito telefônico. Brasília: Ministério da Saúde, 2008. Disponível em: <http:// portal.saude.gov.br/portal/arquivos/pdf/ vigitel2007_final_web.pdf $>$. Acesso em: 10 set. $200 \overline{9}$. 
Vigitel Brasil 2008: vigilância de fatores de risco e proteção para doenças crônicas por inquérito telefônico. Brasília: Ministério da Saúde, 2009.

Vigilância de doenças crônicas não transmissíveis. Brasília, 2011. Disponível em: <http://portal.saude.gov.br/ portal/saude/profissional/visualizar_texto. cfm?idtxt $=31877$ \&janela $=1>$. Acesso em: 03 mar. 2011.

CARVALHAES, M. A. B. L.; MOURA, E. C.; MONTEIRO, C. A. Prevalência de fatores de risco para doenças crônicas: inquérito populacional mediante entrevistas telefônicas em Botucatu, São Paulo, 2004. Revista Brasileira de Epidemiologia, São Paulo, v. 11, n. 1, p.14-23, 2008.

CENTERS FOR DISEASE CONTROL AND PREVENTION - CDC. National diabetes fact sheet.2007. Atlanta: CDC, 2007. Disponível em: <http://www.cdc.gov/diabetes/pubs/pdf/ ndfs_2007.pdf>. Acesso em: 4 out. 2009.

\section{. National Health Interview Survey (NHIS). Atlanta: CDC, 2008.Disponível em: <http://www.cdc.gov/nchs/nhis.htm>. Acesso em: 08 set. 2009. \\ CASADO, L.; VIANNA, L. M.; THULER, L. C. S. Fatores de risco para doenças crô- nicas não transmissíveis no Brasil: uma revisão sistemática. Revista Brasileira de Cancerologia, Rio de Janeiro, v. 55, n. 4, p. 379-388. 2009.}

COSTA, J. D. et al. Consumo abusivo de álcool e fatores associados: estudo de base populacional. Revista de Saúde Pública, Rio de Janeiro, v. 38, n. 2, p. 284-291, 2004.

CUNHA, I. C. et al. Fatores associados à prática de atividade física na população adulta de Goiânia: monitoramento por meio de entrevistas telefônicas. Revista Brasileira de Epidemiologia, São Paulo, v. 11, n. 3, p. 495-504, 2008.

FIGUEIREDO, V. C. Um panorama do tabagismo em 16 capitais brasileiras e Distrito Federal: tendências e heterogeneidades. 2007.166 f. Tese (Doutorado) - Instituto de Medicina Social, Universidade do Estado do Rio de Janeiro, Rio de Janeiro, 2007.
FINE, J. L. Prevalence of multiple chronic disease risk factors 2001 National Health Interview Survey. American Journal of Preventive Medicine, Amsterdam, v. 27, supl. 2, p. 18-24, 2004.

INSTITUTO NACIONAL DE ALIMENTAÇÃO E NUTRIÇÃO (Brasil). Pesquisa Nacional sobre Saúde e Nutrição (PNSN): perfil de crescimento da população brasileira de 0 a 25 anos. Brasília: Secretaria de Projetos Especiais, 1990.

INSTITUTO NACIONAL DE CÂNCER (Brasil). Inquérito domiciliar sobre comportamento de risco e morbidade referida de doenças e agravos não transmissíveis: Brasil, 15 capitais e Distrito Federal, 2002-2003. Rio de Janeiro: Inca, 2004.

LESSA, I. Níveis séricos de creatinina: hipercreatininemia em segmento da população adulta de Salvador, Brasil. Revista Brasileira de Epidemiologia, São Paulo, v. 7, n. 2, p.176-186, 2004.

LETHBRIDGE-ÇEIKU, M.; SCHILLER, J. S.; BERNADEL, L. Summary health statistics for U.S. Adults: National Health Interview Survey. [S.I]: National Center for Health Statistics. Vital Health Stat 10, n. 222, 2002.

MARCOPITO, L. F. et al. Prevalence of a set of risk factors for chronic diseases in the city of São Paulo, Brazil. Revista de Saúde Pública, São Paulo, v. 39, n. 5, p.738-745, out. 2005.

MARTINEZ, T. L. R. et al. Campanha nacional de alerta sobre o colesterol elevado: determinação do nível de colesterol de 81.262 brasileiros. Arquivos Brasileiros de Cardiologia, São Paulo, v. 80, n.6, p.631634, 2003.

MONTEIRO, C. A. et al. Monitoramento de fatores de risco para doenças crônicas por entrevistas telefônicas. Revista de Saúde Pública, São Paulo, v. 39, n. 1, p. 47-57, 2005.

MOURA, E. C. Vigilância de fatores de risco para doenças crônicas por inquérito telefônico nas capitais dos 26 estados brasileiros e no Distrito Federal (2006). Revista Brasileira de Epidemiologia, São Paulo, v. 11, supl. 1, p.20-37, 2008. 
PEIXOTO, M. R. G. et al. Monitoramento por entrevistas telefônicas de fatores de risco para doenças crônicas: experiência de Goiânia, Goiás, Brasil. Cadernos de Saúde Pública, Rio de Janeiro, v. 24, n. 6 , p. 1323-1333, jun. 2008.

PEREIRA, J. C.; BARRETO, S. M.; PASSOS, V. M. O perfil de saúde cardiovascular dos idosos brasileiros precisa melhorar: estudo de base populacional. Arquivos Brasileiros de Cardiologia, São Paulo, v. 91, n. 1, p. 1-10, 2008.

SOCIEDADE BRASILEIRA DE DIABETES. Diretrizes da Sociedade Brasileira de Diabetes 2008. In: SOCIEDADE BRASILEIRA DE DIABETES. Diabetes mellitus pós-transplante. São Paulo, 2009, p. 42-48.

SOCIEDADE BRASILEIRA DE HIPERTENSÃO. 2011. São Paulo. Disponível em <http://www.sbh.org.br>. Acesso em: 02 mar. 2011.

SOUZA, L. J. et al. Prevalência de diabetes mellitus e fatores de risco em Campos dos Goytacazes, RJ. Arquivos Brasileiros de Endocrinologia e Metabologia, São Paulo, v. 47 , n. 1, p. 69-74, fev. 2003

SOUZA,M. F. M. Editorial. Dos dados a política: a importância da informação em
saúde.Epidemiologia e Serviços de Saúde, Brasília, v. 17, n. 1, p. 5-6, jan./mar. 2008.

STATACORP. Stata statistical software: release 8.0. College Station, TX: Stata Corporation, 2003.

THEME FILHA, M. M.; SZWARCWALD, C. L.; SOUZA JUNIOR, P. R. B. Medidas de morbidade referida e inter-relações com dimensões de saúde. Revista de Saúde Pública, São Paulo, v. 42, n. 1, p. 73-81, 2008.

YOKOTA, R. T. C. et al. Prevalência de fatores de risco para doenças crônicas não-transmissíveis em duas regiões do Distrito Federal. Comunicação em Ciências da Saúde, Brasília, v. 18, n. 4, p. 289-296, 2007.

VIACAVA, F. Informações em saúde: a importância dos inquéritos populacionais. Ciência \& Saúde Coletiva, Rio de Janeiro, v. 7, n. 4. p.607-621, 2002.

WORLD HEALTH ORGANIZATION. International guide for monitoring alcohol consumption and related harm. Geneva: WHO, 2000.

World Health Report 2002.

Reducing Risks, Promoting Healthy Life. Geneva: WHO, 2002.

\section{Resumen}

Factores asociados al riesgo para enfermedades no transmisiblesen adultos brasileños: estudio transversal de base poblacional

En Brasil, debido a los cambios en los perfiles demográficos, epidemiológicos y nutricionales de la población, las enfermedades infecciosas hanido cediendo lugar a las crónico-degenerativas. Frente a la gran heterogeneidad demográfica, social y económica del país, se exige nacciones de salud pública que sean dirigidas a las realidades locales. Con el objetivo de identificar los factores asociados al riesgo para enfermedades no transmisibles en adultos brasileños, se realizó un estudio domiciliario, entre junio de 2002 y octubre de 2005, consistente en una muestra representativa de 18 capitales brasileñas. Las prevalencias de los factores de riesgo para enfermedades crónicas no-transmisibles fueron: exceso de peso; actividad física irregular o sedentarismo; consumo de cigarrillos; consumo de alcohol de riesgo; hipertensión arterial declarada; diabetes declarada; e hipercolesterolemia declarada. La ausencia de factores de riesgo fue observada en solamente un $8,5 \%$ de la población entrevistada. Asípues, se concluye que la alta prevalencia de exposición a por lo menos uno de los factores de riesgo estudiados 
evidencia que las acciones en salud deben ser multifactoriales y no dirigidas exclusivamente a un factor aislado.

Palabras-clave: Factores de riesgo. Enfermedades no transmisibles. Prevalencia. Estudios transversales. Brasil.

\begin{abstract}
Risk factors associated with non-communicable diseases in Brazilian adults: populationbased cross-sectional study
\end{abstract}

Due to changes in the demographic, epidemiological and nutritional profiles of the population, infectious diseases are being replaced by chronic diseasesin Brazil. Public health actions directed toward local scenarios are being required due to the great demographic, social and economic diversity of the country. We performed a household survey aiming to identify the risk factors associated with non-communicable diseases in Brazilian adults. Representative samples were collected from 18 Brazilian state capitals between June 2002 and October 2005. We reported the prevalence for the following risk factors for non-communicable diseases: overweight; irregular physical activity or sedentary; smoking; risk-related alcohol consumption; declared arterial hypertension; declared diabetes mellitus and declared high level of cholesterol. Absence of risk factors was reported only by $8.5 \%$ of the population interviewed. In conclusion, the high prevalence of exposure to at least one of the risk factors studied shows that health actions must be multifactorial and not be directed towarda single isolated factor.

Keywords: Riskfactors. Non-communicable diseases. Prevalence. Cross-sectional studies. Brazil.

Recebido para publicação em 19/07/2011

Aceito para publicação em 05/03/2012 